\title{
TEORES DE CARBONO EM DIFERENTES TIPOS DE PAPEIS
}

\author{
Carlos Roberto Sanquetta ; Paulo Henrique Corrêa Korbela do Rosário²; Ana Paula \\ Dalla Corte ${ }^{1}$; Camila Midori Inoue Sanquetta ${ }^{3}$; Greyce Charllyne Benedet Maas ${ }^{4}$ \\ 1 - Eng. Florestal, Dr., Professor da Universidade Federal do Paraná \\ (carlossanquetta@gmail.com) \\ 2 - Eng. Florestal, Pós-graduando em Manejo Florestal de Precisão, Universidade \\ Federal do Paraná \\ 3 - Arquiteta (autônoma) \\ 4 - Tecnóloga Ambiental, Dra. em Ciências Florestais (autônoma) \\ Av. Lothário Meissner, 900 - Jardim Botânico \\ Curitiba - PR, 80.210-170 - Brasil
}

\section{Recebido em: 06/04/2019 - Aprovado em: 10/06/2019 - Publicado em: 30/06/2019}

DOI: 10.18677/EnciBio_2019A127

\begin{abstract}
RESUMO
Produtos florestais colhidos (PFC) são potencialmente considerados sumidouros de carbono (C), sendo contabilizados em inventários de emissões e remoções de gases de efeito estufa (GEE). Não existem estudos específicos sobre os teores de carbono em papeis produzidos no Brasil, o que conduz ao uso de valores default desenvolvidos em outros países. Este trabalho analisou os teores de C em 252 amostras de diferentes tipos de papeis produzidos no Brasil, sendo: revestido (coated), filtro, guardanapo, higiênico, toalha, de imprimir, jornal, pardo, embalagem e de cigarros. Os papeis foram secos a $70^{\circ} \mathrm{C}$ em estufa e submetidos a análise por combustão a seco. Foram realizados os testes estatísticos de ANOVA e Tukey para comparação. Os teores de C variaram de 26,43 a $47,50 \%$ e houve diferença estatística a $99 \%$ de probabilidade entre os tipos de papeis. As médias dos teores por tipo de papel foram as seguintes: revestido (30,17\%), filtro $(41,93 \%)$, guardanapo $(41,85 \%)$, higiênico $(41,02 \%)$, toalha $(41,48 \%)$, de imprimir $(36,15 \%)$, jornal $(46,46 \%)$, pardo $(41,55 \%)$, embalagem $(38,985)$ e de cigarros $(31,02 \%)$. Papeis com menor grau de processamento industrial apresentaram maiores teores de C. Isso decorre da maior fração de material lenhoso vegetal por unidade de peso. O default do IPCC (Painel Intergovernamental sobre Mudanças Climáticas), de $38,60 \%$, pode ser considerado adequado para alguns papeis, mas não para a maioria. Concluiu-se que, ao realizar inventários de emissões de GEE, o Brasil para contabilizar as emissões e remoções em papeis utilize os teores de $\mathrm{C}$ deste este estudo, por serem mais adequados à realidade do País.
\end{abstract}

PALAVRAS-CHAVE: emissões, produtos florestais, sumidouro

\section{CARBON CONTENT IN DIFFERENT PAPER TYPES}

\begin{abstract}
Harvested wood products (HWP) are potentially considered carbon sinks (C) and are accounted for in inventories of greenhouse gas emissions and removals (GHG). There are no specific studies on the carbon content of papers produced in Brazil,
\end{abstract}


which leads to default values developed in other countries. This work analyzed the $\mathrm{C}$ contents in 252 samples of different types of papers produced in Brazil, being: coated, filter, napkin, toilet, towel, printing, newspaper, brown, packaging and cigarettes. Papers were dried at $70^{\circ} \mathrm{C}$ in an oven and subjected to dry combustion analysis. Statistical ANOVA and Tukey tests were performed for comparison. The $\mathrm{C}$ contents ranged from 26.43 to $47.50 \%$ and there was statistical difference at $99 \%$ probability among paper types. The average contents of paper were as follows: coated $(30.17 \%)$, filter $(41.93 \%)$, napkin $(41.85 \%)$, toilet $(41.02 \%)$, towel), printing $(36.15 \%)$, newspaper $(46.46 \%)$, brown $(41.55 \%)$, packaging $(38,985)$ and cigarettes $(31.02 \%)$. Papers with lower industrial processing degree presented higher levels of $\mathrm{C}$. This is due to the higher fraction of woody material per unit weight. The IPCC (Intergovernmental Panel on Climate Change) default of $38.60 \%$ may be considered adequate for some papers, but not for the majority. It was concluded that, in carrying out GHG emissions inventories to account for emissions and removals in paper, Brazil uses the $\mathrm{C}$ levels of this study, as they are more appropriate to the country's reality.

KEYWORDS: emissions, forest products, sink

\section{INTRODUÇÃO}

Produtos florestais colhidos (PFC), do inglês harvested wood products (HWP), entre os quais o papel, são potencialmente considerados reservatórios de carbono, podendo gerar emissões ou remoções de GEE (IPCC, 2003). Eles desempenham um papel importante na mitigação climática (KAYO et al., 2015, YANG; ZANG, 2016), cujas emissões por fontes ou remoções por sumidouro devem ser incluídas nos inventários nacionais de GEE após o segundo período de compromisso do Protocolo de Quioto (IPCC, 2014).

Apesar do seu papel em armazenar $C$, se constituindo num sumidouro, PFC também podem se converter em fonte de emissões de GEE, na medida que ocorre a sua decomposição (IPCC, 2006). O Brasil ainda não contempla nos seus inventários de GEE as emissões e remoções por PFC, nem como sumidouro nem como fonte de emissão (MCTI, 2016).

A quantificação de PFC é contemplada no volume 12 das Diretrizes do IPCC (2006) sobre inventários nacionais de GEE e prevê distintas abordagens e metodologias. Em todas elas, é necessário saber o teor de $\mathrm{C}$, também chamado de fração de $\mathrm{C}$, ou ainda fator de conversão de $\mathrm{C}$ em alguns dos guias metodológicos.

Existem muitos estudos sobre teores de $C$ na biomassa de espécies florestais, sobretudo na madeira (e.g. DALLAGNOL et al., 2011; BEHLING et al., 2014; WATZLAWICK et al., 2014; SILVA et al., 2014; SANQUETTA et al., 2016) e alguns em materiais de construção (FREITAS JR et al., 2015; FREITAS JR et al., 2018), porém há poucas publicações no tema em PFC e especificamente sobre diferentes tipos de papeis. Ademais, nas tabelas default do IPCC constam valores agregados, derivados de vários tipos de papeis analisados em outros países. Não há publicação relevante sobre a matéria no Brasil que analise as variações dos teores de $\mathrm{C}$ em distintos tipos de papeis.

Este estudo teve como objetivo apresentar as variações nos teores de $\mathrm{C}$ em 10 diferentes tipos de papeis fabricados e utilizados no País, contemplando embalagens, produtos de higiene e uso doméstico, papeis de impressão e cigarros. Almeja-se com esta publicação demonstrar que o teor de $C$, considerado muitas vezes único e homogêneo, apresenta variações e que o uso de valores específicos para cada tipo auxilia na melhoria da qualidade dos inventários de GEE. 


\section{MATERIAL E MÉTODOS}

Diferentes tipos de papeis foram considerados neste estudo. Foram analisadas 252 amostras de 10 tipos de papeis, a saber: revestido (coated), filtro, guardanapo, higiênico, toalha, de imprimir, jornal, pardo, embalagem e de cigarros. A quantidade de amostras por tipo de papel foi variável em função da disponibilidade de material adequado para as análises. Descrição das amostras de papel de cigarros é apresentada em Sanquetta e Anater (2016). Todos os papeis estavam livres de tintas e impressões que poderiam alterar os resultados.

Os papeis foram secos a $70^{\circ} \mathrm{C}$ em estufa, triturados e moídos ao nível de graduação (mesh) 20, compatível com o equipamento analisador. As amostras foram pesadas e introduzidas em cadinhos de porcelana e, então, levadas à análise dos teores de $\mathrm{C}$ por combustão a seco no equipamento marca LECO, modelo C144. O aparelho determina o tempo de combustão e o teor de $C$ da amostra e envia as informações diretamente para um software em computador acoplado, gerando um arquivo digital pronto para as análises estatísticas.

Foi realizada a análise estatística descritiva dos dados. Os dados foram também analisados pelos testes estatísticos de ANOVA para verificar a existência de diferença estatística entre os teores de $\mathrm{C}$ das amostras e do teste Tukey para discriminar as diferenças pareadas entre os tratamentos (tipos de papel). Ambos os testes foram realizados ao nível de $99 \%$ de probabilidade.

\section{RESULTADOS E DISCUSSÕES}

A média geral dos teores de $C$ de todas as amostras foi de $36,39 \%$, ou 0,3639 $\mathrm{MgC} . \mathrm{Mg}^{-1}$, segundo a nomenclatura do IPCC (2006). O valor default agregado do teor de C para papeis é $38,60 \%$. Os teores de C das 252 amostras variaram de 26,43 a 47,50\%, o que representa uma amplitude de 58,06\% em relação à média e um coeficiente de variação de $15,20 \%$ (Tabela 1). Isso demonstra que existem variações expressivas entre os teores de $C$ de papeis produzidos e utilizados no Brasil. Isso decorre da matéria prima utilizada e do processo de fabricação desses papeis, que são diferenciados. Tais variações têm implicações importantes, pois pequenas diferenças no valor de teor de $\mathrm{C}$ aplicado nos cálculos têm grandes repercussões nos inventários, às vezes, implicando em milhões de toneladas de GEE.

TABELA 1. Teores de carbono (\%) em diferentes tipos de papeis fabricados no Brasil

\begin{tabular}{lcccccc}
\hline Tipo de Papel & $\mathrm{n}$ & Média & Mínimo & Máximo & Desvio Padrão & CV\% \\
\hline Revestido & 16 & 30,17 & 27,52 & 31,83 & 1,50 & 4,97 \\
Cigarro & 100 & 31,02 & 26,43 & 34,07 & 1,47 & 4,73 \\
Imprimir & 16 & 36,15 & 34,22 & 38,78 & 1,37 & 3,79 \\
Embalagem & 32 & 38,98 & 37,22 & 41,97 & 1,11 & 2,84 \\
Higiênico & 20 & 41,02 & 38,34 & 42,74 & 1,41 & 3,44 \\
Toalha & 12 & 41,48 & 40,74 & 42,10 & 0,37 & 0,90 \\
Pardo & 8 & 41,55 & 40,35 & 43,82 & 1,18 & 2,83 \\
Guardanapo & 16 & 41,86 & 40,99 & 42,64 & 0,56 & 1,33 \\
Filtro & 16 & 41,93 & 40,73 & 42,91 & 0,61 & 1,46 \\
Jornal & 16 & 46,46 & 45,67 & 47,50 & 0,56 & 1,21 \\
\hline Geral & 252 & 36,29 & 26,43 & 47,50 & 5,52 & 15,20 \\
\hline
\end{tabular}

Fonte: Os autores (2019). 
Os testes de ANOVA e Tukey revelaram existirem diferenças estatísticas a $99 \%$ de probabilidade entre os tipos de papeis (Tabela 2). As médias dos teores por tipo de papel foram as seguintes: revestido $(30,17 \%)$, filtro $(41,93 \%)$, guardanapo $(41,85 \%)$, higiênico $(41,02 \%)$, toalha $(41,48 \%)$, de imprimir $(36,15 \%)$, jornal $(46,46 \%)$, pardo $(41,55 \%)$, embalagem $(38,985)$ e de cigarros $(31,02 \%)$ (Tabela 2$)$.

TABELA 2. ANOVA dos valores de teores de $C$ de papeis produzidos no Brasil

\begin{tabular}{ccccc}
\hline $\begin{array}{c}\text { Fonte de } \\
\text { Variação }\end{array}$ & $\begin{array}{c}\text { Graus de } \\
\text { liberdade }\end{array}$ & $\begin{array}{c}\text { Soma de } \\
\text { Quadrados }\end{array}$ & $\begin{array}{c}\text { Quadrado } \\
\text { Médio }\end{array}$ & \multirow{2}{*}{ Valor F } \\
\hline Tratamentos & 9 & $7,258.4734$ & 806,4970 & $518.0485^{\star *}$ \\
Resíduo & 242 & 376.7452 & 1,5568 & \\
\hline Total & 251 & $7,635.2186$ & 806,4970 & \\
\hline${ }^{* *}$ valor $p=1,1102 \times 10^{-16}$ & & & \\
Fonte: Os autores $(2019)$. & & &
\end{tabular}

Cinco grupos de tratamentos (tipos de papeis) foram diferenciados pelo teste de Tukey: 1. papeis revestidos e de cigarros, 2. papel de imprimir, 3. papel de embalagem, 4. papeis de uso doméstico e pardo de embrulho, 5. papel jornal (Figura 1). A única média considerada estatisticamente equivalente ao default do IPCC foi a do papel de imprimir. Todas as demais foram diferentes, o que indica que a adoção desse valor pode ser problemática na realização de um inventário de GEE.

Recomenda-se, pois, o uso discriminado do teor de $\mathrm{C}$ por tipo de papel e dentro da realidade nacional, ou seja, com base em análises laboratoriais feitas com papeis fabricados no local de produção, no caso do Brasil para os produtos aqui produzidos e valores do país de origem sendo o papel importado. Para isso é fundamental que o inventário brasileiro de GEE compute as emissões e remoções decorrentes de PFC, em particular do papel, para retratar com maior realismo todos os reservatórios de $\mathrm{C}$ recomendados pelo IPCC. Como o Brasil não importa grandes quantidades de papel atualmente, o uso dos valores publicados neste estudo atende satisfatoriamente a esta recomendação.

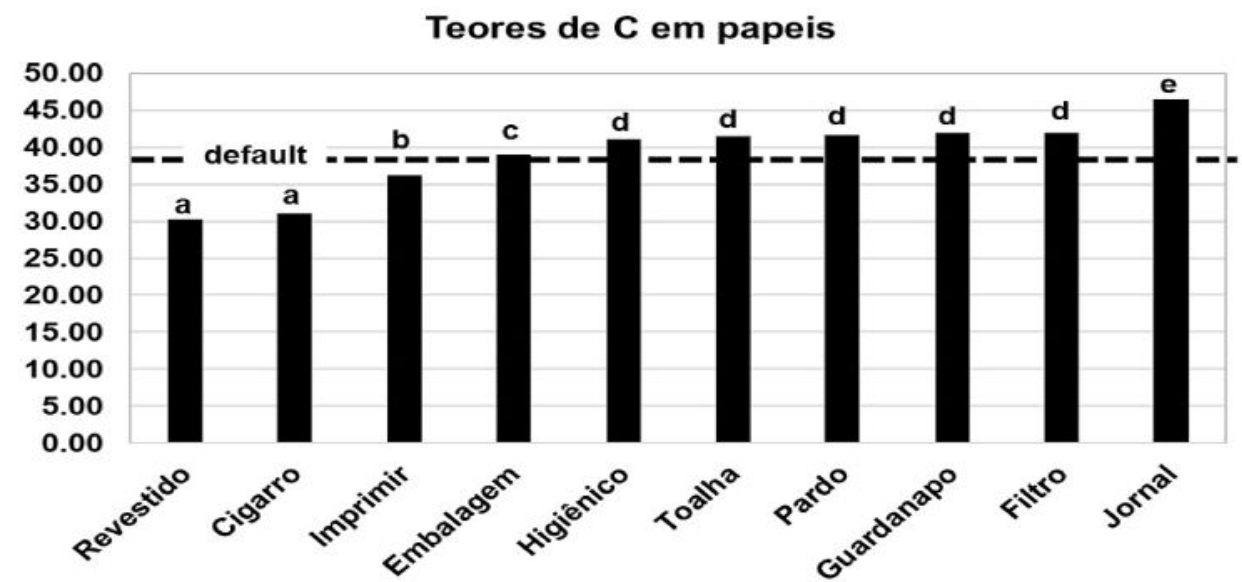

FIGURA 1. Médias dos teores de $C$ em papeis e respectivo teste de Tukey e valor default do IPCC. Médias seguidas pelas mesmas letras não diferem estatisticamente pelo teste de Tukey a 99\% de probabilidade. Fonte: Os autores (2019).

O Brasil é um grande produtor de celulose e papel. Seu consumo interno também vem crescendo, assim como as exportações e importações (FAOSTAT, 2019). O País produz atualmente cerca de 10 milhões de toneladas anuais. De 2004 
a 2014 o crescimento médio na produção de papeis foi de 3\% ao ano, embora nos últimos anos tenha havido uma queda em função da crise econômica. Portanto, o papel é um dos principais PFC produzidos no Brasil. Considerar isso no balanço de $\mathrm{C}$ nos inventários de GEE informados à Convenção-Quadro das Nações Unidas sobre Mudanças Climáticas é muito importante.

Quase a totalidade do papel hoje produzido no Brasil advém de florestas plantadas (IBÁ, 2018). Essas florestas, além de ter a capacidade de fixar na sua biomassa e absorver $\mathrm{CO}_{2}$ via fotossíntese, também podem contribuir com a mitigação climática via estocagem nos PFC (SANQUETTA et al., 2018), como madeira processada mecanicamente, painéis, papel, carvão vegetal, ferro-gusa, etc. O papel dos PFC na mitigação climática tem sido evidenciado em recentes pesquisas (e.g. PILLI et al., 2015; BOUYER; SERENGIL, 2016; BRUNET-NAVARRO et al., 2016; JASINEVIČIUS et al., 2017; ZHANG et al., 2018).

Ao considerar PFC nos inventários de GEE deve-se atentar para as abordagens e metodologias apropriadas recomendadas pelo IPCC. As variáveis fundamentais nessa contabilização são as quantidades produzidas de papel no país, suas importações e exportações, além da massa seca ao ar e o teor de carbono no material (IPCC, 2006). Usar indiscriminadamente o valor default do IPCC para teor de $\mathrm{C}$ é preocupante e torna-se fundamental que níveis de abordagem mais elevados (Tiers) sejam propostos, como recomendado pelo próprio Painel.

Neste estudo foram analisados os teores de $\mathrm{C}$ em diferentes tipos de papeis $\mathrm{e}$ propostos valores alternativos que podem ser usados para melhorar a acurácia e diminuir as incertezas nos futuros inventários de GEE do Brasil.

\section{CONCLUSÕES}

- Os teores de $\mathrm{C}$ dos papeis fabricados no Brasil são distintos do valor default indicado pelo IPCC, exceto o do papel de imprimir;

- Os teores de $\mathrm{C}$ dos distintos tipos de papeis produzidos no Brasil são estatisticamente diferentes;

- O papel que apresentou o maior teor de $C$ foi o jornal, devido ao menor grau de processamento industrial e à matéria prima mais pura em material lenhoso;

- Papeis branqueados com maior grau de processamento industrial possuem menores teores de C;

- É fundamental que as diferenças nos teores de C dos tipos de papeis sejam levadas em conta nos inventários de GEE que computam PFC.

\section{REFERÊNCIAS}

BEHLING, A.; SANQUETTA, C. R.; CARON, B. O.; SCHIMIDT, D.; ELLI, E. F.; DALLA CORTE, A. P. Teores de carbono orgânico de três espécies arbóreas em diferentes espaçamentos. Pesquisa Florestal Brasileira, v. 34, n. 77, p. 13-19, 2014. Disponível em: <https://doi.org/10.4336/2014.pfb.34.77.562> doi: 10.4336/2014.pfb.34.77.562

BOUYER, O.; SERENGIL, Y. Carbon stored in harvested wood products in Turkey and projections for 2020. Journal of the Faculty of Forestry Istanbul University, v. 66, n. 1, p. 295-302, 2016. Disponível em: <http://dx.doi.org/10.17099/jffiu.48603> 
doi: $10.17099 /$ jffiu.48603

BRUNET-NAVARRO, P.; JOCHHEIM, H.; MUYS, B. Effect of cascade use on the carbon balance of the German and European wood sectors. Global Change Biology, v. 22, p. 2555-2569, 2016. Disponível em: <https://doi.org/10.1111/gcb.13235> doi: 10.1111/gcb.13235

DALLAGNOL, F. S.; MOGNON, F.; SANQUETTA, C. R.; DALLA CORTE, A. P. Teores de carbono de cinco espécies florestais e seus compartimentos. Floresta e Ambiente, v. 18, n. 4, p. 410-416, 2011. Disponível em: <http://dx.doi.org/10.4322/floram.2011.060> doi: 10.4322/floram.2011.060

FAO - Food and Agriculture Organization of the United Nations. FAOSTAT. 2019. Disponível em: <http://www.fao.org/faostat/en/\#data/GF>

FREITAS JR, J. A.; SANQUETTA, C. R.; SANQUETTA, M. N. I.; DALLA CORTE, A. P.; SANQUETTA, F. T. I. Estocagem em carbono em portas internas de madeira de edifícios residenciais. Enciclopédia Biosfera, v.11 n.22; p. 1182-1193, 2015. Disponível em: <http://dx.doi.org/10.18677/Enciclopedia_Biosfera_2015_152> doi: 10.18677/Enciclopedia_Biosfera_2015_152

FREITAS JR, J. A.; SANQUETTA, C. R.; IWAKIRI, S.; COSTA, M. R. M. M. F. T. I. The use of wood construction materials as a way of carbon storage in residential buildings in Brazil. International Journal of Construction Management, publicado online, 1-7, 2018. Disponível em: < https://doi.org/10.1080/15623599.2018.1532384> doi: 10.1080/15623599.2018.1532384

IBÁ - INSTITUTO BRASILEIRO DE ÁRVORES. Relatório 2018. São Paulo: IBÁ, 2017. Disponível em : <https://iba.org/datafiles/publicacoes/relatorios/digitalsumarioexecutivo-2018.pdf>

IPCC - Intergovernmental Panel on Climate Change. Good Practice Guidance for Land Use, Land-Use Change and Forestry. IPCC National Greenhouse Gas Inventories Programme, UNEP (2003). Disponível em: <https://www.ipccnggip.iges.or.jp/public/gpglulucf/gpglulucf.html>

IPCC - Intergovernmental Panel on Climate Change. Climate Change. 2006 IPCC Guidelines for National Greenhouse Gas Inventories. Chapter 12 HWP. Disponível em: <https://www.ipccnggip.iges.or.jp/public/2006gl/pdf/4_Volume4/V4_12_Ch12_HWP.pdf>

IPCC - Intergovernmental Panel on Climate Change. Climate Change. 2013 Revised Supplementary Methods and Good Practice Guidance Arising from the Kyoto Protocol. Task Force on National Greenhouse Gas Inventories, 2014. Disponível em: <https://www.ipcc-nggip.iges.or.jp/public/kpsg/index.html>

JASINEVIČIUS, G.; LINDNER, M.; CIENCIALA, E.; TYKKYLÄINEN, M. Carbon accounting in harvested wood products: assessment using material flow analysis resulting in larger pools compared to the IPCC default method. Journal of Industrial Ecology, v. 22, n. 1, p. 121-131, 2017. Disponível em: <https://doi.org/10.1111/jiec.12538> doi: 10.1111/jiec.12538 
KAYO, C.; TSUNETSUGU, Y.; TONOSAKI, M. Climate change mitigation effect of harvested wood products in regions of Japan. Carbon Balance and Management, v. 10, n. 24, publicado online, 2015. Disponível em: <https://dx.doi.org/10.1186\%2Fs13021-015-0036-3> doi: 10.1186\%2Fs13021-0150036-3

MCTI - Ministério da Ciência, Tecnologia e Inovação. III Comunicação Nacional do Brasil à Convenção-Quadro das Nações sobre Mudanças Climáticas. Vol. 3, 336p. 2016.

PILLI, R.; FIORESE, G.; GRASSI, G. EU mitigation potential of harvested wood products. Carbon Balance and Management, 10, n. 6. Publicado online. 2015. Disponível em: <https://doi.org/10.1186/s13021-015-0016-7> doi: 10.1186/s13021015-0016-7

SANQUETTA, C. R.; ANATER, M. J. N. Emissões de $\mathrm{CO}_{2}$ e geração de resíduos pelo consumo de cigarros no Brasil no período 2006-2012. BIOFIX Scientific Journal, v. 1, n. 1, p. 33-37, 2016. Disponível em: <http://dx.doi.org/10.5380/biofix.v1i1.49097> doi: 10.5380/biofix.v1i1.49097

SANQUETTA, C. R.; DALLA CORTE, A. P.; PELISSARI, A. L.; TOMÉ, M.; MAAS, G. C. B.; SANQUETTA, M. N. I. Dynamics of carbon and $\mathrm{CO}_{2}$ removals by Brazilian forest plantations during 1990-2016. Carbon Balance and Management, v. $13 \mathrm{n}$. 20, p. 1-12, 2018. Disponível em: <https://doi.org/10.1186/s13021-018-0106-4> doi: 10.1186/s13021-018-0106-4

SANQUETTA, M. N. I.; SANQUETTA, C. R.; MOGNON, F.; DALLA CORTE, A. P.; MAAS, G. C. B. Wood density and carbon content in young teak individuals from Pará, Brazil. Científica, v. 44, n. 2, p. 608-614, 2016. Disponível em: http://dx.doi.org/10.15361/1984-5529.2016v44n4p608-614>

SILVA, S. A.; DALLA CORTE, A. P.; SANQUETTA, C. R.; RODRIGUES, A. L.; BARRETO, T. G. Teores de carbono médios para compartimentos e espécies florestais. Enciclopédia Biosfera, v. 10, n. 19, p. 2990-3007, 2014.

WATZLAWICK, L. F.; MARTINS, P. J.; RODRIGUES, A. L.; EBLING, A. A.; BALBINOT, R.; LUSTOSA, S. B. C. Teores de carbono em espécies da floresta ombrófila mista e efeito do grupo ecológico. Cerne, v. 20, n. 4, p. 613-620, 2014. Disponível em: <http://dx.doi.org/10.1590/01047760201420041492> doi: $10.1590 / 01047760201420041492$

YANG H.; ZANG X. A rethinking of the Production Approach in IPCC: its objectiveness in China. Sustainability, 8, n. 216, publicado online, 2016. Disponível em: < https://doi.org/10.3390/su8030216> doi: 10.3390/su8030216

ZHANG, X.; YANG, H.; CHEN, J. Life-cycle carbon budget of China's harvested wood products in 1900-2015. Forest Policy and Economics, v. 92, p. 181-192 2018. Disponível em: <https://doi.org/10.1016/j.forpol.2018.05.005> doi: 10.1016/j.forpol.2018.05.005 